Vietnam Journal of Mechanics, VAST, Vol.39, No. 4 (2017), pp. 351 -364

DOI:10.15625/0866-7136/9810

\title{
NONLINEAR THERMO-MECHANICAL STABILITY OF SHEAR DEFORMABLE FGM SANDWICH SHALLOW SPHERICAL SHELLS WITH TANGENTIAL EDGE CONSTRAINTS
}

\author{
Nguyen Minh Khoa ${ }^{1}$, Hoang Van Tung ${ }^{2, *}$ \\ ${ }^{1}$ University of Transport Technology, Ha Noi, Viet Nam \\ ${ }^{2}$ Hanoi Architectural University, Viet Nam \\ *E-mail: hoangtung0105@gmail.com \\ Received April 25, 2017
}

\begin{abstract}
This paper presents an analytical approach to investigate the nonlinear axisymmetric response of moderately thick FGM sandwich shallow spherical shells resting on elastic foundations, exposed to thermal environments and subjected to uniform external pressure. Material properties are assumed to be temperature independent, and effective properties of FGM layer are graded in the thickness direction according to a simple power law distribution in terms of the volume fractions of the constituents. Formulations are based on first order shear deformation shell theory taking geometrical nonlinearity, initial geometrical imperfection, Pasternak type elastic foundations and varying degree of tangential constraint of boundary edge into consideration. Approximate solutions are assumed to satisfy clamped boundary condition and Galerkin method is applied to derive closed-form expressions of critical buckling loads and nonlinear load-deflection relation. Effects of geometrical parameters, thickness of face sheets, foundation stiffness, imperfection, thermal environments and degree of tangential edge constraints on the nonlinear stability of FGM sandwich shallow spherical shells are analyzed and discussed.
\end{abstract}

Keywords: Functionally graded material, shallow spherical shell, sandwich shell, nonlinear stability, tangential edge constraint.

\section{INTRODUCTION}

Structural elements in the form of spherical shells are widely used in many engineering structures. Since these shells are usually exposed to complex loading conditions, their static and dynamic responses are important problems and received considerable attention. Static and dynamic responses of isotropic spherical shells have been investigated in works [1-6] using analytical and semi-analytical approaches. Subsequently, axisymmetric bucking and post-buckling behavior of moderately thick shallow spherical shells

(C) 2017 Vietnam Academy of Science and Technology 
made of symmetrically laminated composites and subjected to uniform external pressure have been analyzed by $\mathrm{Xu}$ [7] utilizing a Fourier-Bessel series solution and by Nath and Sandeep [8] using an iterative Chebyshev series solution technique. Also, Muc [9] made use of trigonometric series method to consider unsymmetrical buckling and postbuckling behavior of laminated shallow spherical shells subjected to external pressure.

The appearance of functionally graded materials (FGMs) necessitates more studies on static and dynamic responses of shallow spherical shells made of these novel materials. Shahsiah and his co-workers $[10,11]$ used an analytical method on the basis of adjacent equilibrium criterion to investigate linear asymmetric buckling of simply supported FGM shallow and deep spherical shells under thermal loads. Based on an alternative approach, Bich and Tung [12] investigated the nonlinear axisymmetric response of clamped thin FGM shallow spherical shells under uniform external pressure taking the effects of pre-existent thermal loads and initial imperfection into consideration. They made use of the classical shell theory to establish equilibrium and compatibility equations in terms of deflection and stress functions and these equations are solved by applying Galerkin method. This work is then extended by Boroujerdy and Eslami [13] for piezo-FGM shallow spherical shells, and by Duc et al. [14] for FGM shallow spherical shells on elastic foundations. Basing on an analytical method, nonlinear unsymmetrical static and dynamic buckling behavior of FGM shallow spherical shells have been analyzed by Bich et al. [15]. Recently, Tung [16] used equilibrium equations in terms of displacements and rotation on the basis of the first order shear deformation shell theory to study the nonlinear thermo-mechanical response of FGM shallow spherical shells. Actually, foregoing works only considered two extreme cases of in-plane boundary conditions as boundary edge is assumed to be unrestrained (movable edge) or fully restrained (immovable edge). In practical situations, edges of plate and shell may be partially movable only, and tangential edge constraints have considerable and important effects on the nonlinear stability and load carrying capacity of plate and shell structures [17]. Recently, Tung [18,19] considered the effects of tangential edge constraints on the post-buckling of thin FGM cylindrical panels and shear deformable FGM sandwich plates under thermal and mechanical loads. More recently, Tung analyzed separate and simultaneous influences of elastic foundations and tangential constraints of edges on the nonlinear stability of FGM shallow spherical shells [20]. Some studies relating to stability of FGM plate and cylindrical shells on elastic foundations, buckling of FGM conical panel and postbuckling of FGM shallow spherical shell panel under nonuniform thermal environment have been addressed in works [21-24] basing on some approaches.

Motivated by previous works [18-20] and from lack of results for FGM sandwich spherical shells, this paper presents an analytical approach to investigate the nonlinear axisymmetric response of shear deformable FGM sandwich shallow spherical shells resting on elastic foundations, exposed to thermal environments and mechanically loaded by uniform external pressure. Approximate solutions are assumed to satisfy clamped boundary condition and Galerkin method is applied to obtain closed-form expressions of critical buckling loads and load-deflection relation. Numerical illustrations show the 
effects of geometrical parameters, thickness of face sheets, foundation stiffness, geometrical imperfection, thermal environments and degree of tangential edge constraint on the nonlinear stability of FGM sandwich shallow spherical shells.

\section{FGM SANDWICH SHALLOW SPHERICAL SHELL (FGM SSSS) ON AN ELASTIC FOUNDATION}

Consider a Sandwich Shallow Spherical Shell (SSSS) of radius of curvature $R$, base radius $a$, uniform total thickness $h$ and rise of shell $H$. The shell is clamped at boundary edge, rested on a Pasternak elastic foundation and is defined in a coordinate system $(\varphi, \theta, z)$ whose origin is located on the middle surface of the shell, $\varphi$ and $\theta$ are in the meridional and circumferential directions, respectively, and $z$ is perpendicular to the middle surface and points inwards $(-h / 2 \leq z \leq h / 2)$ as shown in Fig. 1 .

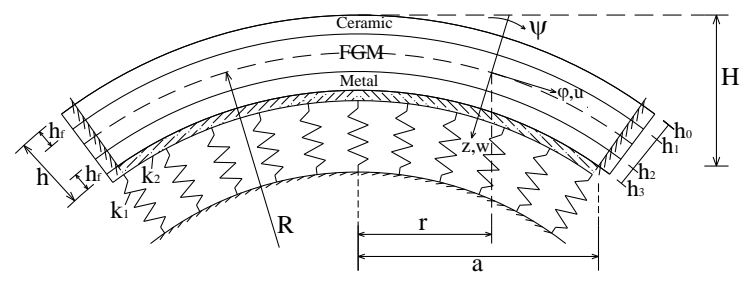

Fig. 1. Configuration and coordinate system of a sandwich shallow spherical shell (SSSS) on an elastic foundation

The SSSS is constructed from two isotropic face sheets (i.e. skins) separated by a thicker core layer made of FGMs. It is assumed that core layer and face sheets are perfectly bonded and the thickness of each face sheet is $h_{f}$. The top skin is isotropic homogeneous (fully ceramic) and the bottom skin is also isotropic homogeneous (fully metal), whereas FGM core layer is graded from a ceramic-rich interface to a metal-rich interface. This type of FGM SSSS is asymmetric about the mid-plane and the volume fraction of metal phase is expressed as

$$
\begin{aligned}
& V_{m}(z)=0, z \in\left[h_{0}, h_{1}\right], \text { top skin } \\
& V_{m}(z)=\left(\frac{z-h_{1}}{h_{2}-h_{1}}\right)^{n}, z \in\left[h_{1}, h_{2}\right], \text { core layer } \\
& V_{m}(z)=1, z \in\left[h_{2}, h_{3}\right], \text { bottom skin }
\end{aligned}
$$

Herein $h_{0}=-\frac{h}{2}, h_{1}=-\frac{h}{2}+h_{f}, h_{2}=\frac{h}{2}-h_{f}, h_{3}=\frac{h}{2}$ and $n \geq 0$ is volume fraction index that defines the gradation of material properties across the thickness direction. Moreover, the volume fraction of the ceramic constituent is given as $V_{c}(z)=1-V_{m}(z)$.

Effective properties of FGM SSSS such as modulus of elasticity $E$ and coefficient of thermal expansion $\alpha$ can be determined by the linear rule of mixture as

$$
\begin{aligned}
& E(z)=E_{c}+E_{m c} V_{m}(z), \\
& \alpha(z)=\alpha_{c}+\alpha_{m c} V_{m}(z),
\end{aligned}
$$


where

$$
E_{m c}=E_{m}-E_{c}, \quad \alpha_{m c}=\alpha_{m}-\alpha_{c}
$$

and $E_{m}, \alpha_{m}\left(E_{c}, \alpha_{c}\right)$ are temperature independent properties of metal (ceramic) constituents. Poisson's ratio $v$ is assumed to be constant in the present study.

\section{FORMULATIONS}

In the present study, the first order shear deformation shell theory is used for formulation and the FGM SSSS is assumed to be under axisymmetric deformation. According to this theory, displacement components $\bar{u}, \bar{v}, \bar{w}$ in $\varphi, \theta, z$ directions, respectively, at a distance $z$ from the middle surface are represented as [7]

$$
\bar{u}(r, z)=u(r)+z \psi(r), \quad \bar{v}(r, z)=0, \quad \bar{w}(r, z)=w(r),
$$

in which $r=R \sin \varphi, u$ is displacement in the meridional direction at the middle surface, $w$ is the deflection of the shell, and $\psi$ is the rotation of a normal to the middle surface. Due to shallowness of the shell, it is approximately assumed that $\cos \varphi=1, R d \varphi=d r$ and $R=a^{2} /(2 H)$. The non-zero strain components of the shell are defined as

$$
\varepsilon_{r}=\varepsilon_{r 0}+z \chi_{r}, \quad \varepsilon_{\theta}=\varepsilon_{\theta 0}+z \chi_{\theta}, \quad \varepsilon_{r z}=\psi+w_{, r},
$$

where a comma denotes differentiation with respect to the corresponding variable, i.e. ()$_{, r}=d() / d r$, and the strains at the middle surface $\varepsilon_{r 0}, \varepsilon_{\theta 0}$ and curvatures $\chi_{r}, \chi_{\theta}$ are related to the displacements and rotation in the form

$$
\varepsilon_{r 0}=u_{, r}-w / R+w_{, r}^{2} / 2, \quad \varepsilon_{\theta 0}=u / r-w / R, \quad \chi_{r}=\psi_{, r}, \quad \chi_{\theta}=\psi / r .
$$

Based on Hooke's law, stress-strain relations for an FGM SSSS including temperature effects are

$$
\begin{aligned}
\sigma_{r} & =\frac{E(z)}{1-v^{2}}\left[\varepsilon_{r}+v \varepsilon_{\theta}-(1+v) \alpha(z) \Delta T\right], \\
\sigma_{\theta} & =\frac{E(z)}{1-v^{2}}\left[\varepsilon_{\theta}+v \varepsilon_{r}-(1+v) \alpha(z) \Delta T\right], \\
\sigma_{r z} & =\frac{E(z)}{2(1+v)} \varepsilon_{r z},
\end{aligned}
$$

where $\Delta T$ denotes the change of environment temperature from thermal stress free initial state. The force and moment resultants are expressed in terms of the stress components through the thickness as

$$
\left(N_{r}, N_{\theta}\right)=\int_{-h / 2}^{h / 2}\left(\sigma_{r}, \sigma_{\theta}\right) d z, \quad\left(M_{r}, M_{\theta}\right)=\int_{-h / 2}^{h / 2}\left(\sigma_{r}, \sigma_{\theta}\right) z d z, \quad Q_{r}=K_{S} \int_{-h / 2}^{h / 2} \sigma_{r z} d z,
$$

where $K_{S}$ is shear correction coefficient. 
Introduction of Eqs. (5) and (7) into Eqs. (8), the force and moment resultants are rewritten in the form

$$
\begin{aligned}
& {\left[N_{r}, M_{r}\right]=\frac{\left[E_{1}, E_{2}\right]}{1-v^{2}}\left(\varepsilon_{r 0}+v \varepsilon_{\theta 0}\right)+\frac{\left[E_{2}, E_{3}\right]}{1-v^{2}}\left(\chi_{r}+v \chi_{\theta}\right)-\frac{\left[\Phi_{0}, \Phi_{1}\right]}{1-v},} \\
& {\left[N_{\theta}, M_{\theta}\right]=\frac{\left[E_{1}, E_{2}\right]}{1-v^{2}}\left(\varepsilon_{\theta 0}+v \varepsilon_{r 0}\right)+\frac{\left[E_{2}, E_{3}\right]}{1-v^{2}}\left(\chi_{\theta}+v \chi_{r}\right)-\frac{\left[\Phi_{0}, \Phi_{1}\right]}{1-v},} \\
& Q_{r}=\frac{K_{S} E_{1}}{2(1+v)}\left(\psi+w_{, r}\right),
\end{aligned}
$$

where

$$
\left[\Phi_{0}, \Phi_{1}\right]=\int_{-h / 2}^{h / 2}[1, z] E(z) \alpha(z) \Delta T d z
$$

and

$$
\begin{aligned}
& E_{1}=E_{c} h+E_{m c}\left(h_{f}+\frac{h-2 h_{f}}{n+1}\right), \\
& E_{2}=\frac{E_{m c}}{2}\left[h h_{f}-h_{f}^{2}+\frac{n}{(n+1)(n+2)}\left(h-2 h_{f}\right)^{2}\right] \\
& E_{3}=\frac{E_{c} h^{3}}{12}+E_{m c}\left[\frac{1}{4} h^{2} h_{f}-\frac{1}{2} h h_{f}^{2}+\frac{1}{3} h_{f}^{3}+\frac{\left(h-2 h_{f}\right)^{3}}{n+1}\left(\frac{1}{4}-\frac{1}{n+2}+\frac{2}{(n+2)(n+3)}\right)\right] .
\end{aligned}
$$

The nonlinear equilibrium equations of the geometrically imperfect FGM SSSS resting on elastic foundations are [20]

$$
\begin{aligned}
& D\left(r \psi_{, r r r}+2 \psi_{, r r}-\frac{\psi, r}{r}+\frac{\psi}{r^{2}}\right)+\frac{r}{R} \Delta_{s} f+f_{, r r}\left(w_{, r}+w_{, r}^{*}\right) \\
& +f_{, r}\left(w_{, r r}+w_{, r r}^{*}\right)+r\left(q-q_{f}\right)=0, \\
& D\left(r \psi_{, r r}+\psi, r-\frac{\psi}{r}\right)-\frac{K_{S} E_{1}}{2(1+v)} r\left(\psi+w_{, r}\right)=0 .
\end{aligned}
$$

The strain compatibility equation of the geometrically imperfect FGM SSSS is [20]

$$
\frac{1}{E_{1}} \Delta_{s}^{2} f=-\frac{1}{R} \Delta_{s} w-\frac{1}{r} w_{, r} w_{, r r}-\frac{1}{r} w_{, r} w_{, r r}^{*}-\frac{1}{r} w_{, r}^{*} w_{, r r}
$$

These equations (12a), (12b) and (13) are expressed in terms of variable functions including the deflection $w(r)$, rotation $\psi(r)$ and stress function $f(r)$ defined as

$$
N_{r}=\frac{f_{, r}}{r}, \quad N_{\theta}=f_{, r r} .
$$

In addition, $D$ is flexural rigidity, $\Delta_{S}$ is Laplace operator in case of axisymmetry

$$
D=\frac{E_{1} E_{3}-E_{2}^{2}}{E_{1}\left(1-v^{2}\right)}, \quad \Delta_{s}=\frac{d^{2}}{d r^{2}}+\frac{1}{r} \frac{d}{d r} .
$$


Furthermore, $w^{*}(r)$ is a known function representing initial geometrical imperfection, $q$ is uniform external pressure and $q_{f}$ is shell-foundation interaction represented by Pasternak model as

$$
q_{f}=k_{1} w-k_{2} \Delta_{s} w
$$

in which $k_{1}$ is Winkler foundation modulus and $k_{2}$ is the shear layer foundation stiffness of Pasternak model.

The FGM SSSS is assumed to be clamped on the boundary edge and under axisymmetric deformation. The symmetry condition at the center $r=0$ together out-of-plane and in-plane boundary conditions at $r=a$ are expressed in the form $[4,7,8]$

$$
\begin{aligned}
& \psi=0, N_{r}=f_{, r} / r \text { is finite } \quad \text { at } r=0, \\
& w=0, \psi=0, N_{r}=N_{r 0} \quad \text { at } r=a,
\end{aligned}
$$

where $N_{r 0}$ is fictitious compressive stress due to tangential edge restraint, and $N_{r 0}=0$ for movable clamped edge.

To satisfy boundary conditions (17), approximate mode shapes for the deflection and rotation are assumed as [1]

$$
\left(w, w^{*}\right)=(W, \mu h) \frac{\left(a^{2}-r^{2}\right)^{2}}{a^{4}}, \quad \psi=\Psi \frac{r\left(a^{2}-r^{2}\right)}{a^{3}},
$$

where $W$ is the amplitude of the deflection, and imperfection function $w^{*}$ is assumed to be in the form of deflection for the sake of simplicity. Also, non-dimensional parameter $\mu$ represents imperfection size and $\Psi$ is a coefficient to be determined.

Substitution of $w$ and $w^{*}$ from Eqs. (18) into Eq. (13) and integrating of the resulting equation, at which constants of integration are determined from finiteness condition at the center $r=0$ and in-plane boundary condition $N_{r}(r=a)=N_{r 0}$, yield the stress function as

$$
f_{, r}=\frac{E_{1}}{R a^{4}}\left(\frac{a^{2} r^{3}}{2}-\frac{r^{5}}{6}-\frac{a^{4} r}{3}\right) W-\frac{E_{1}}{a^{8}}\left(a^{4} r^{3}-\frac{2 a^{2} r^{5}}{3}+\frac{r^{7}}{6}-\frac{a^{6} r}{2}\right) W(W+2 \mu h)+N_{r 0} r
$$

Subsequently, introduction of Eqs. (18) into Eq. (12b) and applying Galerkin method for the resulting equation

$$
\int_{0}^{a} L_{1} \times \frac{r\left(a^{2}-r^{2}\right)}{a^{3}} d r=0,
$$

where $L_{1}$ is the resulting expression received after substituting $w$ and $\psi$ from Eqs. (18) into the left-hand side of Eq. (12b). Implementing integration in the Eq. (20a) gives the coefficient $\Psi$ as

$$
\Psi=\frac{4 K_{S} E_{1} a}{32(1+v) D+K_{S} E_{1} a^{2}} W
$$

Now, introduction of Eqs. (18) and (19) into the equilibrium equation (12a) and applying Galerkin method for obtained equation

$$
\int_{0}^{a} L_{2} \times \frac{\left(a^{2}-r^{2}\right)^{2}}{a^{4}} d r=0,
$$


where $L_{2}$ is the resulting expression received after substituting $w, w^{*}$ and $\psi$ from Eqs. (18) into the left-hand side of Eq. (12a). Performing integration in the Eq. (21a) yields the following relation

$$
\begin{aligned}
q= & {\left[\frac{64 \overline{D E}_{1} K_{S}}{R_{a}^{2} R_{h}^{2}\left(32(1+v) \bar{D}+\bar{E}_{1} K_{S} R_{a}^{2} R_{h}^{2}\right)}+\frac{3 \bar{E}_{1}}{7 R_{h}^{2}}+\frac{\bar{D}}{7 R_{a}^{4} R_{h}^{4}}\left(\frac{16}{3} K_{1}+40 K_{2}\right)\right] \bar{W} } \\
& -\frac{976 \bar{E}_{1}}{693 R_{a}^{2} R_{h}^{3}} \bar{W}(\bar{W}+\mu)-\frac{409 \bar{E}_{1}}{693 R_{a}^{2} R_{h}^{3}} \bar{W}(\bar{W}+2 \mu) \\
& +\frac{848 \bar{E}_{1}}{429 R_{a}^{4} R_{h}^{4}} \bar{W}(\bar{W}+\mu)(\bar{W}+2 \mu)+\left[\frac{40}{7 R_{a}^{2} R_{h}^{2}}(\bar{W}+\mu)-\frac{2}{R_{h}}\right] \bar{N}_{r 0},
\end{aligned}
$$

where

$$
\begin{aligned}
& \bar{D}=D / h^{3}, \quad \bar{E}_{1}=E_{1} / h, \quad \bar{E}_{2}=E_{2} / h^{2}, \quad \bar{W}=W / h, \quad \bar{N}_{r 0}=N_{r 0} / h, \\
& R_{a}=a / R, \quad R_{h}=R / h, \quad K_{1}=k_{1} a^{4} / D, \quad K_{2}=k_{2} a^{2} / D .
\end{aligned}
$$

If the transverse shear deformation and foundation interaction are neglected, the expression (21b) leads to result which is similar to relation obtained in the work [12] for thin FGM shallow spherical shell without elastic foundation.

In the present study, the boundary edge of the shell is assumed to be clamped and with varying degrees of tangential restraint. The average end-shortening displacement $\Delta_{r}$ at the boundary edge $r=a$ is related to the corresponding compressive edge load $N_{r 0}$ by

$$
\Delta_{r} c=N_{r 0},
$$

where $c$ is the average tangential stiffness in the meridional direction.

Eq. (23) indicates that value of $\Delta_{r}=0$, enforced by selecting $c \rightarrow \infty$, corresponds to immovable edge, whereas condition of movable edge corresponds to $c=0$ and $N_{r 0}=0$. Also, intermediate degrees of in-plane edge constraint correspond to values of $c$ such that $0<c<\infty$. The expression for the average end-shortening displacement is given by

$$
\Delta_{r}=-\frac{1}{2 \pi a^{2}} \int_{0}^{2 \pi} \int_{0}^{a} u_{, r} r d r d \theta .
$$

By virtue of Eqs. (6), (9) and (14), one can obtain the following relation for imperfect FGM SSSS

$$
u_{, r}=\frac{1}{E_{1}}\left(\frac{f_{, r}}{r}-v f_{, r r}\right)-\frac{E_{2}}{E_{1}} \psi_{, r}-\frac{1}{2} w_{, r}^{2}-w_{, r} w_{, r}^{*}+\frac{w}{R}+\frac{\Phi_{0}}{E_{1}} .
$$

Introduction of Eqs. (18), (19) into Eq. (25) and then substituting the resulting expression into Eq. (24) yield the following relation of the average end-shortening displacement

$$
\begin{aligned}
\Delta_{r}= & {\left[\frac{5 v-7}{72 R}-\frac{K_{S} E_{2}}{32(1+v) D+K_{S} E_{1} a^{2}}\right] W } \\
& +\frac{35-13 v}{144 a^{2}} W(W+2 \mu h)-\frac{(1-v)}{2 E_{1}} N_{r 0}-\frac{\Phi_{0}}{2 E_{1}} .
\end{aligned}
$$


In this study, thermal load is considered for case of uniform temperature rise and temperature change $\Delta T$ is independent of thickness variable. From Eq. (10), thermal parameter $\Phi_{0}$ can be expressed as

$$
\Phi_{0}=P \Delta T h
$$

where

$$
P=E_{c} \alpha_{c}+\left(E_{c} \alpha_{m c}+E_{m c} \alpha_{c}\right)\left(\frac{1-2 \bar{h}_{f}}{n+1}+\bar{h}_{f}\right)+E_{m c} \alpha_{m c}\left(\frac{1-2 \bar{h}_{f}}{2 n+1}+\bar{h}_{f}\right) .
$$

Herein, $\bar{h}_{f}=h_{f} / h$ is face sheet thickness to total thickness ratio.

The combination of Eqs. (23), (26) and (27) gives the fictitious compressive force resultant on tangentially restrained edge $\bar{N}_{r 0}$. Next, substituting the expression of $\bar{N}_{r 0}$ into Eq. (21b) leads to the following explicit expression of nonlinear load-deflection relation

$$
\begin{aligned}
q= & A_{1} \bar{W}-A_{2} \bar{W}(\bar{W}+\mu)-A_{3} \bar{W}(\bar{W}+2 \mu)+A_{4} \bar{W}(\bar{W}+\mu)(\bar{W}+2 \mu) \\
& +\left[\frac{2}{R_{h}}-\frac{40}{7 R_{a}^{2} R_{h}^{2}}(\bar{W}+\mu)\right] e P \Delta T,
\end{aligned}
$$

where

$$
\begin{aligned}
A_{1}= & \frac{64 K_{S} \bar{D} \bar{E}_{1}}{R_{a}^{2} R_{h}^{2}\left(32(1+v) \bar{D}+K_{S} \bar{E}_{1} R_{a}^{2} R_{h}^{2}\right)}+\frac{4 e K_{S} \bar{E}_{1} \bar{E}_{2}}{32(1+v) \bar{D} R_{h}+K_{S} \bar{E}_{1} R_{a}^{2} R_{h}^{3}} \\
& +\frac{3 \bar{E}_{1}}{7 R_{h}^{2}}+\frac{(7-5 v)}{18 R_{h}^{2}} e \bar{E}_{1}+\frac{\bar{D}}{7 R_{a}^{4} R_{h}^{4}}\left(\frac{16}{3} K_{1}+40 K_{2}\right), \\
A_{2}= & \frac{976 \bar{E}_{1}}{693 R_{a}^{2} R_{h}^{3}}+\frac{10(7-5 v)}{63 R_{a}^{2} R_{h}^{3}} e \bar{E}_{1}+\frac{80 K_{S} e \bar{E}_{1} \bar{E}_{2}}{7 R_{a}^{2} R_{h}^{2}\left(32(1+v) \bar{D}+K_{S} \bar{E}_{1} R_{a}^{2} R_{h}^{2}\right)^{\prime}}, \\
A_{3}= & \frac{409 \bar{E}_{1}}{693 R_{a}^{2} R_{h}^{3}}+\frac{(35-13 v)}{36 R_{a}^{2} R_{h}^{3}} e \bar{E}_{1}, \quad A_{4}=\frac{848 \bar{E}_{1}}{429 R_{a}^{4} R_{h}^{4}}+\frac{5(35-13 v)}{63 R_{a}^{4} R_{h}^{4}} e \bar{E}_{1}, \\
e= & \frac{\bar{c}}{2 \bar{E}_{1}+\bar{c}(1-v)}, \quad \bar{c}=c / h .
\end{aligned}
$$

Specialization of Eq. (29) for case of $\Delta T=0$ gives pressure-deflection relation in the form

$$
q=B_{1} \bar{W}-B_{2} \bar{W}^{2}+B_{3} \bar{W}^{3},
$$

where

$$
B_{1}=A_{1}-\mu A_{2}-2 \mu A_{3}+2 \mu^{2} A_{4}, \quad B_{2}=A_{2}+A_{3}-3 \mu A_{4}, \quad B_{3}=A_{4} .
$$

From Eq. (31), values of deflection at extremum points on the pressure-deflection curves may be determined from condition $d q / d \bar{W}=0$ yielding

$$
\bar{W}_{1,2}=\frac{B_{2} \mp \sqrt{B_{2}^{2}-3 B_{1} B_{3}}}{3 B_{3}},
$$


provided

$$
B_{2}^{2}-3 B_{1} B_{3} \geq 0 \text {. }
$$

In case of material and geometrical properties of FGM SSSS, foundation stiffness and degree of in-plane edge constraint make the condition (34) satisfied, the shell experiences an extreme-type buckling behavior which upper and lower buckling pressures may be predicted as $q_{c r}=q\left(\bar{W}_{1}\right)$ and $q\left(\bar{W}_{2}\right)$, respectively. In contrast, extremum-type buckling will not occur and pressure-deflection paths are monotonically developed as $B_{2}^{2}-3 B_{1} B_{3}<0$.

Furthermore, Eq. (29) indicates that pressure-loaded FGM SSSS in thermal environment can exhibit a bifurcation-type buckling behavior and corresponding bifurcation point pressure is predicted as

$$
q_{b}^{\Delta T}=q(\bar{W}=0)=\frac{2}{7 R_{a}^{2} R_{h}^{2}}\left(7 R_{a}^{2} R_{h}-20 \mu\right) e P \Delta T,
$$

which is independent of stiffness of elastic foundation, as $\Delta T$ and tangential stiffness $c$ are not zero-valued. It is interesting from Eq. (29) that for all values of temperature change $\Delta T$, there is existence of an intersection point between load-deflection curves predicted as $\left(\bar{W}^{*}=7 R_{a}^{2} R_{h} / 20-\mu, q\left(\bar{W}^{*}\right)\right)$.

\section{RESULTS AND DISCUSSION}

There are no results in the literature about the subject of the present study for direct comparison. Therefore, the nonlinear axisymmetric response of an isotropic homogeneous shallow spherical shell resting on an elastic foundation and subjected to uniform external pressure is considered as part of verification of the present approach.

Load-deflection curve of a geometrically perfect, immovable clamped shallow spherical shell is depicted in Fig. 2 in comparison with results reported by Civalek [6] using discrete singular convolution and differential quadrature methods. As can be seen, a good agreement is achieved in this comparison.

To illustrate the proposed approach, this section considers FGM SSSSs composed of aluminum $(\mathrm{Al})$ and alumina $\left(\mathrm{Al}_{2} \mathrm{O}_{3}\right)$ with the following properties [12]

$$
\begin{aligned}
& E_{m}=70 \mathrm{GPa}, \quad \alpha_{m}=23 \times 10^{-6}{ }^{\circ} \mathrm{C}^{-1}, \\
& E_{c}=380 \mathrm{GPa}, \quad \alpha_{c}=7.4 \times 10^{-6}{ }^{\circ} \mathrm{C}^{-1},
\end{aligned}
$$

whereas Poisson's ratio is chosen to be $v=0.3$ for both materials. The shear correction coefficient is assumed to be $K_{S}=5 / 6$ in calculation. Moreover, to measure the degree of edge restraint in a convenient way, an alternate tangential stiffness parameter $\beta$ is introduced such that $\beta=0$ and $\beta=1$ correspond to movable and immovable boundary edge $r=a$, respectively, and partially restrained edge is defined by $0<\beta<1$. In the present study, this alternate tangential stiffness parameter is given by

$$
\beta=\frac{\bar{c}}{\bar{E}_{1}+\bar{c}} \text {. }
$$




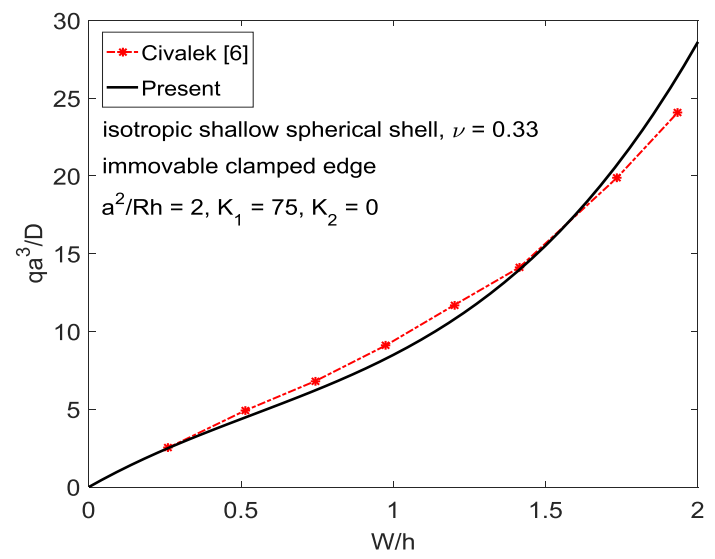

Fig. 2. Comparison of load-deflection response of an isotropic homogeneous shallow spherical shell

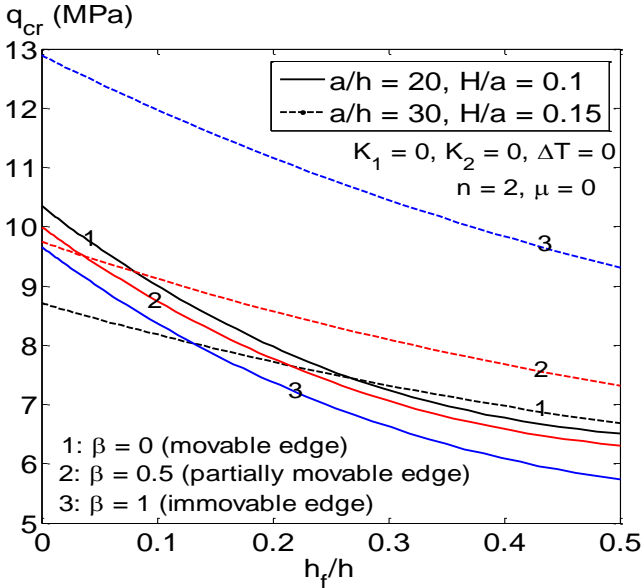

Fig. 3. Effects of $h_{f} / h$ ratio and tangential edge constraints on the critical buckling pressure of FGM SSSSs

In characterizing the behavior of the FGM SSSSs, deformations in which the central region of a shell moves toward the center of respective sphere are referred to as (positive) inward deflections, whereas deformations in the opposite direction referred to as (negative) outward deflection. Since effects of volume fraction index have been mentioned in many studies, this section only considers FGM SSSSs with index $n=2$.

Fig. 3 considers the effects of $h_{f} / h$ ratio and $\beta$ parameter on the critical pressures for $\left(\mathrm{Al}_{2} \mathrm{O}_{3} / \mathrm{FGM} / \mathrm{Al}\right)$ FGM SSSSs under uniform external pressure only. It is evident that the critical buckling pressures are reduced as $h_{f} / h$ ratio to be higher. In addition, the critical loads are enhanced as $a / h$ and $H / a$ ratios are increased and boundary edge is more rigorously restrained in tangential motion, i.e. higher values of $\beta$ parameter.

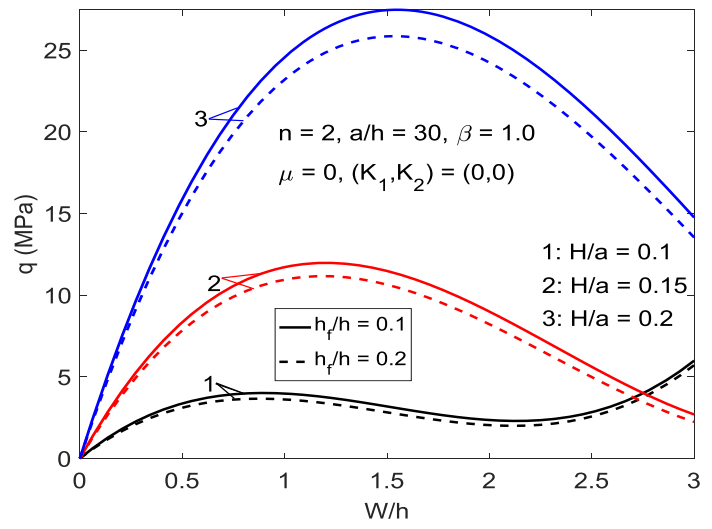

Fig. 4. Effects of $h_{f} / h$ and $H / a$ ratios on the nonlinear response of FGM SSSSs under uniform external pressure

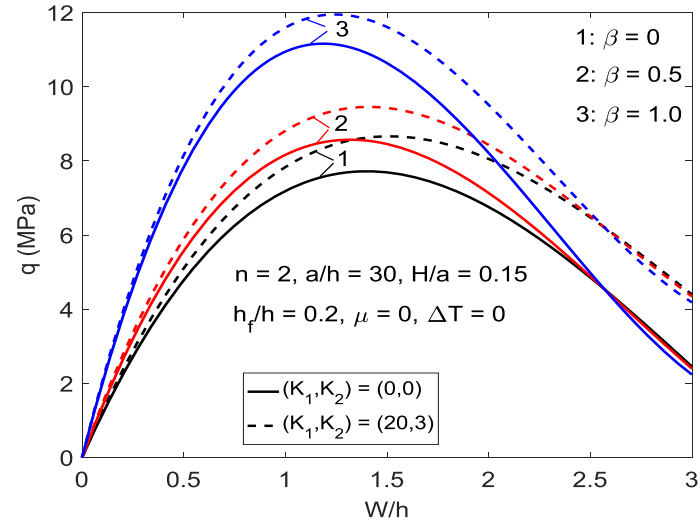

Fig. 5. Effects of tangential edge constraints $(\beta)$ and elastic foundation on the nonlinear response of FGM SSSSs 
The effects of $h_{f} / h$ and $H / a$ ratios on the nonlinear response of immovable clamped FGM SSSSs under uniform external pressure without elastic foundations and thermal environment are analyzed in Fig. 4. As can be seen, extreme type buckling pressures and severity of snap-through phenomenon are both increased as $\mathrm{H} / \mathrm{a}$ ratio become larger (i.e. deeper shell). Furthermore, the pressure-deflection curves become lower with increase in $h_{f} / h$ ratio. Next, Fig. 5 considers the effects of varying degree of tangential edge constraints (i.e. $\beta$ parameter) and elastic foundation on the nonlinear response of FGM SSSSs under uniform external pressure only. Obviously, the increase in value of $\beta$, representing more rigorous constraint of in-plane motion of boundary edge, results in higher buckling loads and more intense snap-through response. In addition, pressure-deflection curves are enhanced and snap-through instability become more benign due to the presence of elastic foundation. Fig. 6 indicates that initial geometrical imperfection has significant influences on the nonlinear response of FGM SSSSs subjected to external pressure. It seems that the effective curvature of the spherical shell is changed due to the presence of initial geometrical imperfection and, as a result, the load carrying capacity of FGM SSSSs is reduced as $\mu$ parameter is increased from -0.1 to 0.1 .

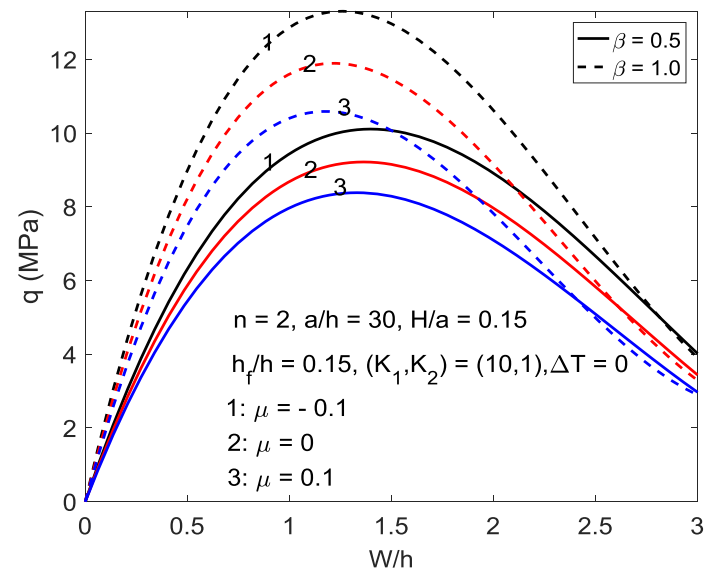

Fig. 6. Effects of tangential edge constraints $(\beta)$ and imperfection $(\mu)$ on the nonlinear response of FGM SSSSs

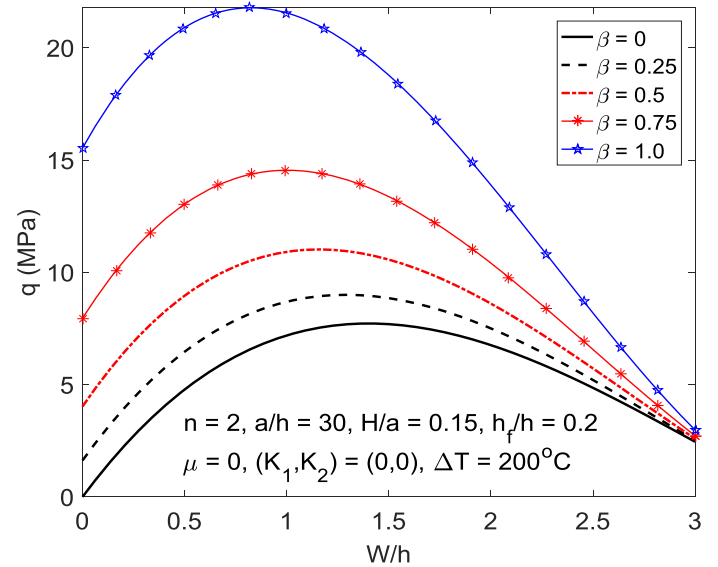

Fig. 7. Effects of tangential edge constraints and thermal environment on nonlinear thermo-mechanical response of FGM SSSSs

The effects of degree of tangential edge constraint and thermal environment on the nonlinear thermo-mechanical response of FGM SSSSs are analyzed in Fig. 7. It is evident that, externally pressurized FGM SSSSs exposed to a thermal environment will exhibit a bifurcation type buckling behavior as boundary edge is tangentially restrained. Moreover, bifurcation point pressure is enhanced by increase in degree of tangential edge constraint. This may be explained that the spherical shell is deflected outward (negative deflection) and, as a result, the shell is more curved due to pre-existent thermal stress, especially as boundary edge is rigorously restrained. Therefore, external pressure must reach a bifurcation point value at which the shell surface returns reference state prior deflecting inward as external pressure exceeds bifurcation point value. 
Subsequently, the effects of thermal environments on the nonlinear thermomechanical response of FGM SSSSs on Pasternak foundations are analyzed in Fig. 8. This figure indicates that bifurcation point pressures are enhanced as $\beta$ parameter and/or temperature change $\Delta T$ are increased. Furthermore, difference between pressure-deflection curves in the two cases of $\beta=0.5$ and $\beta=1.0$ is substantially bigger as FGM SSSSs are exposed to higher temperature fields.

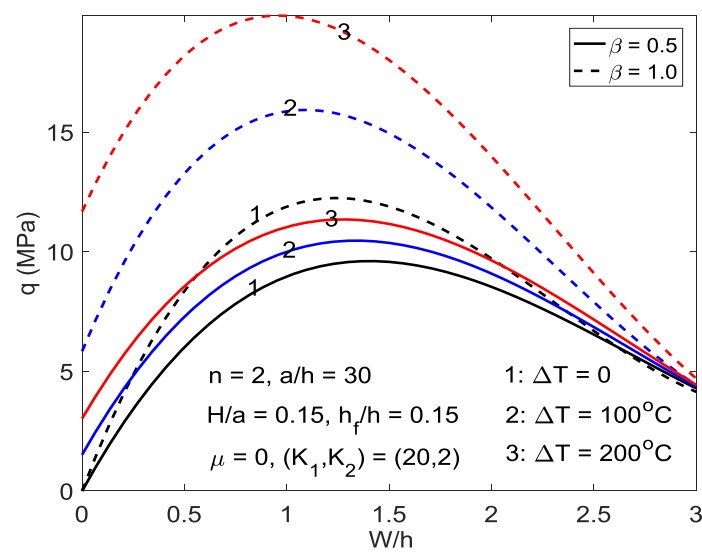

Fig. 8. Effects of tangential stiffness $\beta$ and thermal load on nonlinear thermo-mechanical response of FGM SSSSs

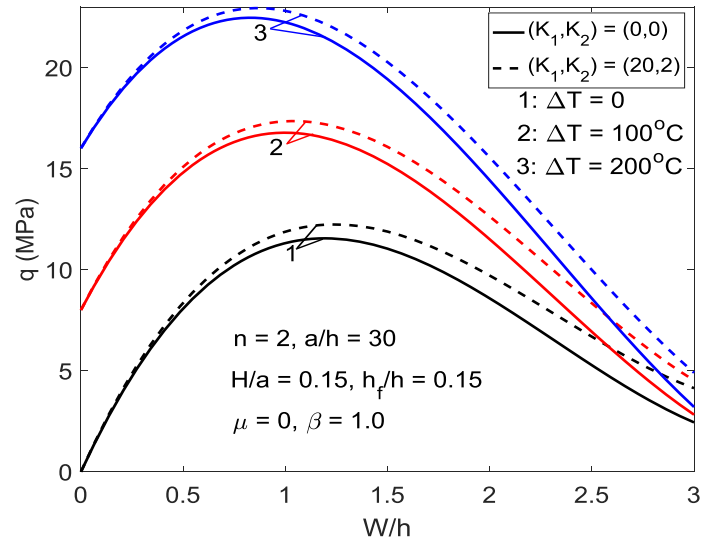

Fig. 9. Effects of elastic foundations and thermal environments on nonlinear thermomechanical response of FGM SSSSs

Finally, Fig. 9 examines the effects of elastic foundations on the thermo-mechanical response of the FGM SSSSs with different values of environment temperature. It is evident that load-deflection curves are enhanced and snap-through phenomenon becomes more benign, i.e. postbuckling response is more stable, when the FGM SSSSs are supported by Pasternak elastic foundations

\section{CONCLUDING REMARKS}

Snap-through instability and the nonlinear response of pressure-loaded FGM SSSSs in thermal environments have been investigated. Formulations are based on the first order shear deformation shell theory and closed-form expressions are derived by applying Galerkin method. The study reveals that thickness of face sheets, geometrical parameters and degree of tangential edge constraint have sensitive effects on the behavior and load carrying capacity of the FGM SSSSs. The shell has higher critical buckling load and more severe snap-through intensity as boundary edge is more rigorously restrained in tangential motion. The results also indicate that elastic foundations have beneficial influences on the load carrying capacity (i.e. higher load-deflection curves) and stabilization (i.e. more benign snap-through response) of the FGM SSSSs. Furthermore, initial geometrical imperfection changes the curvature of spherical shell and has significant effects on the nonlinear stability of the shell. As a final remark, buckling pattern of pressureloaded FGM SSSS is changed from extremum type to bifurcation type as environment 
temperature is elevated and bifurcation point pressure is enhanced with increasing in temperature change $\Delta T$ and/or degree of tangential edge constraint $\beta$.

\section{ACKNOWLEDGEMENT}

This research is funded by Vietnam National Foundation for Science and Technology Development (NAFOSTED) under grant number 107.02-2017.11.

\section{REFERENCES}

[1] M. Sathyamoorthy. Vibrations of moderately thick shallow spherical shells at large amplitudes. Journal of Sound and Vibration, 172, (1), (1994), pp. 63-70. doi:10.1006/jsvi.1994.1158.

[2] M. R. Eslami, H. R. Ghorbani, and M. Shakeri. Thermoelastic buckling of thin spherical shells. Journal of Thermal Stresses, 24, (12), (2001), pp. 1177-1198. doi:10.1080/014957301753251746.

[3] R. Shahsiah and M. R. Eslami. Thermal and mechanical instability of an imperfect shallow spherical cap. Journal of Thermal Stresses, 26, (7), (2003), pp. 723-737. doi:10.1080/713855992.

[4] Q. S. Li, J. Liu, and J. Tang. Buckling of shallow spherical shells including the effects of transverse shear deformation. International Journal of Mechanical Sciences, 45, (9), (2003), pp. 15191529. doi:10.1016/j.ijmecsci.2003.09.020.

[5] G. H. Nie. Asymptotic buckling analysis of imperfect shallow spherical shells on non-linear elastic foundation. International Journal of Mechanical Sciences, 43, (2), (2001), pp. 543-555. doi:10.1016/s0020-7403(99)00118-6.

[6] Ö. Civalek. Geometrically nonlinear dynamic and static analysis of shallow spherical shell resting on two-parameters elastic foundations. International Journal of Pressure Vessels and Piping, 113, (2014), pp. 1-9. doi:10.1016/j.ijpvp.2013.10.014.

[7] X. Chang-Shi. Buckling and post-buckling of symmetrically laminated moderately-thick spherical caps. International Journal of Solids and Structures, 28, (9), (1991), pp. 1171-1184. doi:10.1016/0020-7683(91)90110-2.

[8] Y. Nath and K. Sandeep. Postbuckling of symmetrically laminated, moderately thick, axisymmetric shallow spherical shells. International Journal of Mechanical Sciences, 35, (11), (1993), pp. 965-975. doi:10.1016/0020-7403(93)90033-q.

[9] A. Muc. Buckling and post-buckling behaviour of laminated shallow spherical shells subjected to external pressure. International Journal of Non-Linear Mechanics, 27, (3), (1992), pp. 465-476. doi:10.1016/0020-7462(92)90013-w.

[10] R. Shahsiah, M. R. Eslami, and R. Naj. Thermal instability of functionally graded shallow spherical shell. Journal of Thermal Stresses, 29, (8), (2006), pp. 771-790. doi:10.1080/01495730600705406.

[11] R. Shahsiah, M. R. Eslami, and M. S. Boroujerdy. Thermal instability of functionally graded deep spherical shell. Archive of Applied Mechanics, 81, (10), (2011), pp. 1455-1471. doi:10.1007/s00419-010-0495-7.

[12] D. H. Bich and H. V. Tung. Non-linear axisymmetric response of functionally graded shallow spherical shells under uniform external pressure including temperature effects. International Journal of Non-Linear Mechanics, 46, (9), (2011), pp. 1195-1204. doi:10.1016/j.ijnonlinmec.2011.05.015.

[13] M. S. Boroujerdy and M. R. Eslami. Axisymmetric snap-through behavior of Piezo-FGM shallow clamped spherical shells under thermo-electro-mechanical loading. International Journal of Pressure Vessels and Piping, 120, (2014), pp. 19-26. doi:10.1016/j.ijpvp.2014.03.008. 
[14] N. D. Duc, V. T. T. Anh, and P. H. Cong. Nonlinear axisymmetric response of FGM shallow spherical shells on elastic foundations under uniform external pressure and temperature. European Journal of Mechanics-A/Solids, 45, (2014), pp. 80-89. doi:10.1016/j.euromechsol.2013.11.008.

[15] D. H. Bich, D. Van Dung, and L. K. Hoa. Nonlinear static and dynamic buckling analysis of functionally graded shallow spherical shells including temperature effects. Composite Structures, 94, (9), (2012), pp. 2952-2960. doi:10.1016/j.compstruct.2012.04.012.

[16] H. V. Tung. Nonlinear thermomechanical stability of shear deformable FGM shallow spherical shells resting on elastic foundations with temperature dependent properties. Composite Structures, 114, (2014), pp. 107-116. doi:10.1016/j.compstruct.2014.04.004.

[17] L. Librescu, W. Lin, M. P. Nemeth, and J. H. Starnes Jr. Thermomechanical postbuckling of geometrically imperfect flat and curved panels taking into account tangential edge constraints. Journal of Thermal Stresses, 18, (4), (1995), pp. 465-482. doi:10.1080/01495739508946314.

[18] H. V. Tung. Postbuckling behavior of functionally graded cylindrical panels with tangential edge constraints and resting on elastic foundations. Composite Structures, 100, (2013), pp. 532 541. doi:10.1016/j.compstruct.2012.12.051.

[19] H. V. Tung. Thermal and thermomechanical postbuckling of FGM sandwich plates resting on elastic foundations with tangential edge constraints and temperature dependent properties. Composite Structures, 131, (2015), pp. 1028-1039. doi:10.1016/j.compstruct.2015.06.043.

[20] H. V. Tung. Nonlinear axisymmetric response of FGM shallow spherical shells with tangential edge constraints and resting on elastic foundations. Composite Structures, 149, (2016), pp. 231-238. doi:10.1016/j.compstruct.2016.04.032.

[21] H.-S. Shen. Postbuckling of shear deformable FGM cylindrical shells surrounded by an elastic medium. International Journal of Mechanical Sciences, 51, (5), (2009), pp. 372-383. doi:10.1016/j.ijmecsci.2009.03.006.

[22] Ö. Civalek. Nonlinear dynamic response of laminated plates resting on nonlinear elastic foundations by the discrete singular convolution-differential quadrature coupled approaches. Composites Part B: Engineering, 50, (2013), pp. 171-179. doi:10.1016/j.compositesb.2013.01.027.

[23] Ç. Demir, K. Mercan, and Ö. Civalek. Determination of critical buckling loads of isotropic, FGM and laminated truncated conical panel. Composites Part B: Engineering, 94, (2016), pp. 110. doi:10.1016/j.compositesb.2016.03.031.

[24] V. R. Kar and S. K. Panda. Postbuckling analysis of shear deformable FG shallow spherical shell panel under nonuniform thermal environment. Journal of Thermal Stresses, 40, (1), (2017), pp. 25-39. doi:10.1080/01495739.2016.1207118. 